\title{
Optimizing Geographic Routing for Millimeter-wave Wireless Networks with Directional Antenna
}

\author{
(Invited Paper) \\ L. X. Cai ${ }^{1}$, H. Y. Hwang ${ }^{1}$, X. Shen ${ }^{1}$, J. W. Mark ${ }^{1}$, and L. Cai ${ }^{2}$ \\ 1. Department of Electrical \& Computer Engineering, University of Waterloo, Waterloo, ON, N2L 3G1, Canada \\ 2. Department of Electrical \& Computer Engineering, University of Victoria, BC, V8W 3P6, Canada
}

\begin{abstract}
Millimeter-wave (mmWave) communication technologies can achieve up to several gigabit/sec data rate over a small range, using directional antenna. To enable high data rate wireless connectivity in a large area, a multi-hop routing protocol is needed. The rate-adaptiveness of $\mathrm{mmWave}$ link and the use of directional antenna brings both challenges and opportunities for routing protocol design. In this paper, we propose an Optimal Geographic Routing Protocol (OGRP) and an accompanying distributed directional medium access control (MAC) protocol. We further study how to optimize the selection of relays to maximize the flow throughput and minimize the network resources used by a flow, considering the MAC overheads. By balancing the number of hops and the per hop throughput, the proposed OGRP outperforms the existing greedy geographic routing and opportunistic routing protocols.
\end{abstract}

\section{INTRODUCTION}

The FCC has recently allocated the $57-64 \mathrm{GHz}$ millimeterwave (mmWave) band for general use, which opens a door for very high data rate wireless applications over the 7 $\mathrm{GHz}$ unlicensed band. mmWave communications have main characteristics of short wavelength, large bandwidth and severe oxygen absorption and atmospheric attenuation. Because of the unique characteristics of mmWave communications, it is highly desirable to use directional antenna to achieve much higher antenna gain and transmission range, by directing transmission energy toward the desired direction only. Using mmWave technologies for personal and local area networks has been actively pursued in the literature and by standard bodies, such as the IEEE 802.15.3c and IEEE 802.11 VHT working groups.

Although mmWave communication can achieve up to several gigabit/sec data rate, the transmission ranges and coverage are very limited. In public hotspots where high data rate wireless connectivity in a larger area is needed (e.g., in an airport, expo room, inside an airplane), a networking solution is necessary so data can be relayed over multi-hop wireless paths to reach the destination.

Geographic routing is a promising solution for wireless ad hoc networks, thanks to the low complexity of the localized routing algorithm, scalability with the network size, and fast route convergence when network topology changes. Existing geographic routing algorithms usually select a relaying node based on the geographic distance or direction information, e.g., choose a node with position closest to the destination. In a dense wireless network, the hop count of a greedy geographic routing algorithm nearly matches that of the shortest path algorithm [1]. However, with the shortest path (in terms of hops), the transmission distance in each hop may be long that decreases the flow throughput due to high path loss in mmWave channel. On the other hand, high path loss and the use of directional antenna should allow a more efficient spectrum reuse which can significantly improve the network throughput. The routing protocol design for multihop mmWave networks with directional antenna is an open, challenging problem, which motivates this work.

The main contributions of this paper are three-fold. First, we design a geographic routing protocol for mmWave wireless networks using directional antenna. The next hop relay is selected in a distributed manner, based on the locations of the sender, the destination and the potential relays. Second, we present a contention-based MAC protocol to facilitate the routing with directional antenna. Third, we further study how to optimize the selection of relays to maximize the flow throughput and to minimize the wireless resources taken by the flow, considering the protocol overheads in the MAC layer. Numerical results show that the proposed Optimal Geographic Routing Protocol (OGRP) can outperform the existing geographic routing or opportunistic routing protocols.

The rest of the paper is organized as follows. Section II introduces the related work. Section III presents the system model and introduces the directional MAC protocol. The OGRP protocol is introduced in Section IV. Then we study the protocol performance and how to optimize the protocol parameter in Section V. Numerical results are given in Section VI, followed by the concluding remarks in Section VII.

\section{RELATED WORK}

Geographic routing has been shown to be more scalable than traditional ad hoc routing protocols, because the nodes make simple routing decisions based on the local geographic information of its neighboring nodes and the routing complexity grows with the density of the network rather than the size of the network [2]. With the advances of localization technologies, many geographic routing protocols have been proposed in the literature.

The most-forward-within-radius routing protocol was presented in [3], in which the packet is forwarded to a neighbor with the greatest progress. They show that there is a trade-off between progress and transmission success. A simple greedy geographic routing protocol was proposed in [4], in which a node transmits data to its neighbor closest to the destination. How to improve geographic routing to ensure end-to-end QoS 
was proposed in [5]. In [6], the compass routing protocol was studied and the packets are forwarded to the neighbor such that the direction to the neighbor is closest to direction to the destination. These geographic routing schemes can achieve very high delivery rates in a dense network and the hop counts nearly match that of the shortest path algorithm. However, the greedy schemes may fail in a sparse network with routing holes [1]. GPSR [7] and GOAFR [8] were proposed to overcome the routing hole problem by switching to a boundary tracing scheme until geographic forwarding is successful. The throughput capacity and delay performance of some geographic routing schemes are studied in [9], and a geographic forwarding based random routing algorithm was proposed to achieve high throughput even in networks with routing holes.

Other routing metrics for choosing the best relaying nodes in localized routing protocols have also been studied. A power efficient routing strategy was presented in [10]. The Nearest Closer (NC) method was proposed to forward data to the nearest neighbor to minimize the energy consumption per packet [11]. Some recent routing protocols exploited cooperative diversity of wireless channel to improve flow throughput. In [12], both Rayleigh fading and nonfading channels have been considered for data forwarding. The candidate relaying nodes return acknowledgments in a sequence and the first one who responds is selected as the relay. A distributed non cooperative routing scheme was proposed in [13] to achieve cooperative diversity gain among multiple receivers by selecting the receiver with the highest received signal-tonoise ratio (SNR) as the relay. In wireless networks where the signal power decreases significantly over distance, the node closer to the source usually has a higher SNR. Selecting the nearest node may result in a large number of hops, which may not be desirable in ad hoc networks using a contention-based MAC protocol, because the increased contention level may decrease the network throughput.

Using the high precision localization capability of ultrawideband (UWB) communications [14], geographic routing and efficient MAC design in UWB networks were proposed [15], [16]. By adapting the link rate according to the transceiver distance, the proposed geographic routing protocol and MAC protocol can improve the UWB network throughput. An integrated strategy for MAC and routing in UWB networks was proposed in [17], which exploited the distance information for reducing the power emission and multi user interference in UWB networks, and used a power aware routing metric defined in [10]. Different from the UWB system operating in the $3.1-10.6 \mathrm{GHz}$ frequency band, mmWave UWB systems in the $57-64 \mathrm{GHz}$ band suffer from severe path loss due to oxygen absorption and atmosphere attenuation. Thus, it is essential to use directional antenna to combat the severe path loss for mmWave communications. Due to severe path loss, neither the traditional greedy geographic routing nor the NC method can achieve high throughput in mmWave networks. Therefore, we need to design a geographic routing algorithm to choose proper relaying nodes in mmWave networks. In addition, the use of directional antenna in mmWave networks makes the route discovery more challenging. Although link layer protocol design for mmWave WPANs has been an active area [18], [19], how to jointly explore geographic routing and MAC protocol design in mmWave ad hoc networks using directional antenna is still an open issue.

\section{SYSTEM MODEL}

We consider a dense mmWave wireless network where the nodes may rely on multi-hop relay to reach the destination (such as a gateway to the Internet). For mmWave transmissions, directional antennas are used to achieve higher link data rate and better spatial reuse. For ad hoc networks, it is desirable to let the nodes sense/receive omni-directionally during their idle time, which can mitigate the deafness problem and avoid complicated MAC protocol design. Thus, we assume that each node uses directional antenna for transmitting and receiving packets. When it is idle, it switches on all beams in order to overhear ongoing control messages from all directions. This is possible if each node is equipped with a number of beams, and switch on/off the beams to work in a directional or omni-directional mode. As the size of mmWave antennas is very small and the signal processing capability increases, it is feasible to deploy multiple antenna beams.

\section{A. Directional DCF $M A C$}

The omni-directional receiving during the idle time allows us to use a simple, distributed MAC protocol for the multihop network. Here, we adopt the CSMA/CA based IEEE 802.11 Distributed Coordination Function (DCF) protocol as the baseline, which has been standardized in [20]. To work with directional antenna, the DCF protocol is extended as a directional DCF protocol as follows. First, we need to extend the Network Allocation Vector (NAV) to a Directional Network Allocation Vector (DNAV) [21], so each node keeps a NAV for each direction/beam. For data transmission, if the beam towards the receiver is not blocked by the DNAV, the sending node first senses the channel directionally and then sends a Directional Ready-to-Send (DRTS) message to the receiver. If the receiver receives the DRTS successfully and the receiving beam is not blocked by the receiver's DNAV, it then replies with a Directional Clear-to-Sent (DCTS) message. When any neighboring nodes overhear the DRTS/DCTS messages, they should update their DNAVs to block the corresponding beams accordingly. Once the DRTS/DCTS are exchanged successfully, the sending nodes can send out data packets and wait for the acknowledgment (ACK). Both the data and ACK are transmitted and received directionally.

\section{B. Link model}

With $7 \mathrm{GHz}$ bandwidth, mmWave transceivers use adaptive and diversity technologies (e.g., adaptive modulation and coding) to adapt the link data rate according to the received signal-to-interference-and-noise ratio (SINR), as proposed in the IEEE 802.15.3c and IEEE 802.11 VHT protocols.

The received signal may suffer path loss and time-varying fading and shadowing. Since the mobility is typically low for 


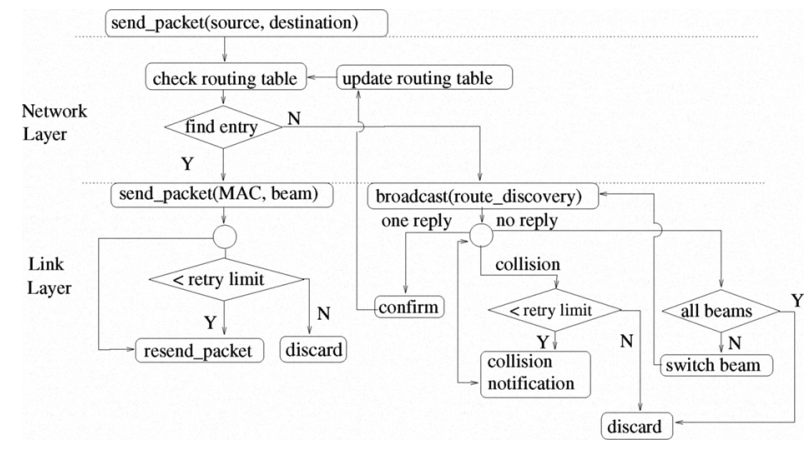

(a) Sender side

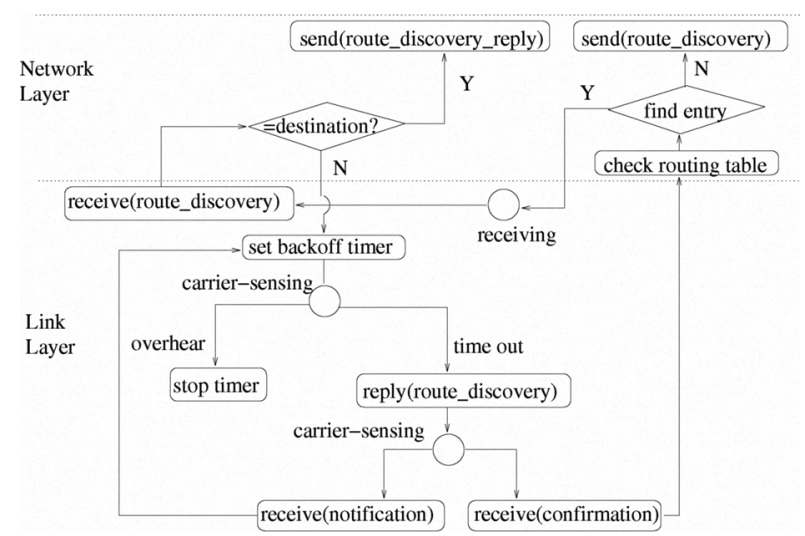

(b) Receiver side

Fig. 1. Interactions between the network and link layers

mmWave networks, the channel quality is relatively stable. As a starting point, we assume the received signal strength is mainly affected by path loss, and our approach can be extended to consider other channel impairments. We model the pass loss $P L$ as:

$$
P L(x)=P L_{0}+\alpha * 10 \log _{10} x \mathrm{~dB},
$$

where $x$ is the transceiver distance, $P L_{0}$ is the path loss (in $\mathrm{dB}$ ) at reference distance $d_{0}=1 \mathrm{~m}$, and $\alpha$ is the path loss exponent.

\section{Optimal Geographic Routing Protocol}

The proposed Optimal Geographic Routing Protocol (OGRP) selects appropriate multi-hop relays from the source to the destination, aiming to maximize the flow throughput and minimize the wireless resources used for the whole path. We assume that each node knows its own location and the source node knows the location of the destination. As it is desired to shorten the latency for path establishment, the proposed routing protocol requires additional assistance from the MAC layer. The main functionality and interactions between the network layer and the MAC layer protocols for sending and receiving a Route_Discovery message are given in Fig. 1.

In a nut shell, when a sender needs to find a path to a destination, the network layer will send a Route_Discovery message using the broadcast service of the MAC layer. The Route_Discovery message contains the network addresses and physical locations of the source and the destination, and also lists the directions that the message can be sent to. When the

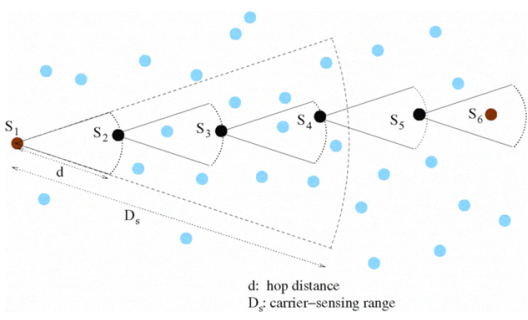

Fig. 2. Multi-hop path

channel is sensed idle, the Route_Discovery message is sent out. After the neighboring nodes receive the Route_Discovery message, they will contend to serve as the relay by reply a Relay_Contention message after a backoff time. The backoff time for Relay_Contention message is different from that for DCTS. If there are collisions for the Relay_Contention messages, the sender sends a Collision_Notification message and let the contending nodes resolve collisions till one relay is selected. The sender then notifies all contending nodes the selected relay using a Relay_Confirmation message. The details of the contention scheme will be given in Section IV-C.

Once a relaying node is selected, the sender will update its routing table with the new entry associated with an expiration time. If the routing table of the relaying node does not have a valid entry of the destination, it broadcasts a Route_Discovery message. This procedure repeats till the destination is reached. Once the path is established, data packets from the source to the destination will be relayed hop by hop with the directional DCF MAC protocol.

In the following subsections, we discuss the major components of the proposed protocols: the sending, receiving, contention, and path repairment procedures.

\section{A. Sending procedure}

With the directional transmissions, for each destination in the routing table, not only the network address but also the corresponding direction (or the node's beam towards that direction) should be recorded. That is, when a packet needs to be transmitted, in addition to the source and destination network addresses, the network layer should let the link layer know which direction the packet should be sent out. For multicast and broadcast messages, the network layer may specify a list of directions (beams) that the messages should be sent out and in what order.

When a node is idle, it uses the all beams for receiving omni-directionally, so the deafness problem is mitigated. When the node overhears any control messages (DRTS/DCTS), it updates its DNAV to block the beam which has received the control messages.

Once a path is established, packet-forwarding by each hop just uses the directional DCF protocol introduced in Section III-A. Here, we focus attention on how the path can be established. With a geographic routing protocol, each node selects the next-hop relay based on the location information, and the end-to-end path is established hop-by-hop, as shown in Fig. 2. The procedure for a node to find the next-hop relay is given in Algorithm 1, and the corresponding procedure for a node to compete for serving as a relay is given in Algorithm 2. 


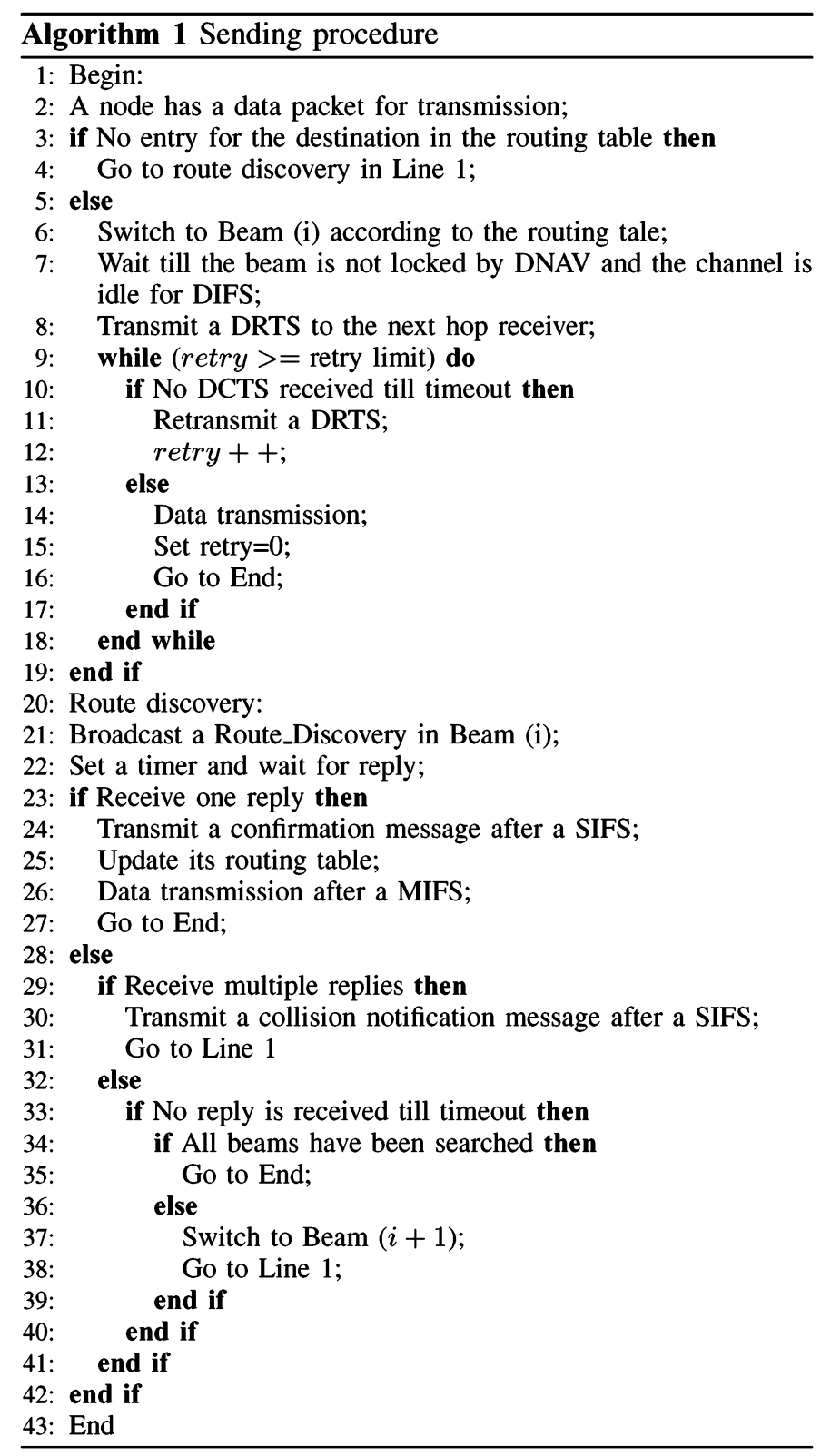

Using Algorithm 1, the route discovery procedure is executed when a node needs to send out a packet and cannot find a valid entry in the routing table for the destination. The proposed OGRP routing protocol first identifies the suitable directions to find the next hop relay or the destination. For instance, for the source node in Fig. 2 with the beam pattern shown in Fig. 3, it sets a prioritized beam list (Beams $1,2, . ., 5)$, and requests the link layer to broadcast the Route_Discovery message using these beams sequentially till a next-hop relay is found. Then, the node updates its routing table and transmits the packet to the selected relay. DIFS, SIFS, and MIFS in the algorithm are constant inter-frame spaces used in the MAC protocol [22].

\section{B. Receiving procedure}

Each broadcast Route_Discovery message may be received by multiple nodes, and we encounter the dilemma for choosing the ideal relay. If the hop distance is too small, the end-to-end

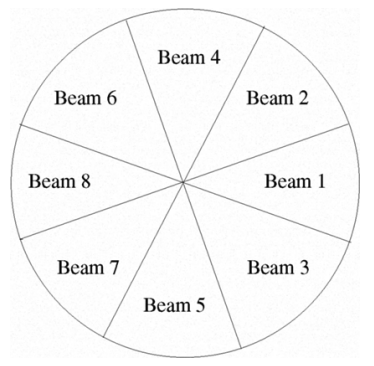

Fig. 3. Beam pattern

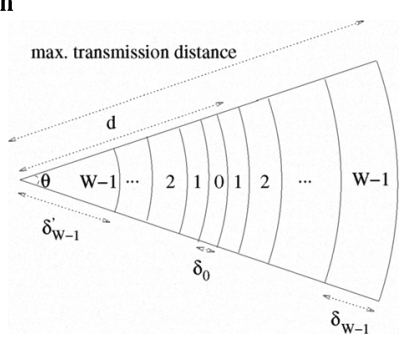

Fig. 4. Contention zones

path may contain many hops and results in high intra-flow contentions; if the hop distance is too large, the SINR for each hop drops quickly and so does the per-hop link throughput. How to identify the optimal location of the relay will be discussed in Section V. Given the optimal hop-distance $d$, we can devise the contention procedure to find appropriate relays in a distributed manner.

As listed in Algorithm 2, when receiving a Route_Discovery message, each contending (non-destination) node will calculate a backoff time (detailed introduction on how to map the location information to the backoff time will be given in the following subsection). If more than one nodes set the same backoff value and their responses are collided, the Route_Discovery sender will send a notification message, so the colliding nodes will choose a random backoff time and contend again, till only one node succeeds in the contention. Thereafter, the Route_Discovery sender will send a confirmation message.

If a node is chosen as the relay, it will check whether it has a valid entry to the flow destination. If not, it will broadcast a Route_Discovery message using Algorithm 1.

\section{Contention scheme}

Ideally, the node with the optimal relay distance $d$ should have a higher priority to serve as the relay. However, besides the location information of the sender and itself, each node has very limited knowledge of the network topology and user deployment. Therefore, we need to design a distributed algorithm to choose the best relay. Here, we use a distributed contention based scheme, which requires a minor modification on the MAC protocol.

Specifically, when a node receives a Route_Discovery message, it calculates a contention backoff timer. If during the backoff it overhears any response from other nodes, it will give up the contention. Otherwise, it sends a reply message to the sender once the backoff timer expires. To avoid potential collisions between the delayed reply frames and transmissions 


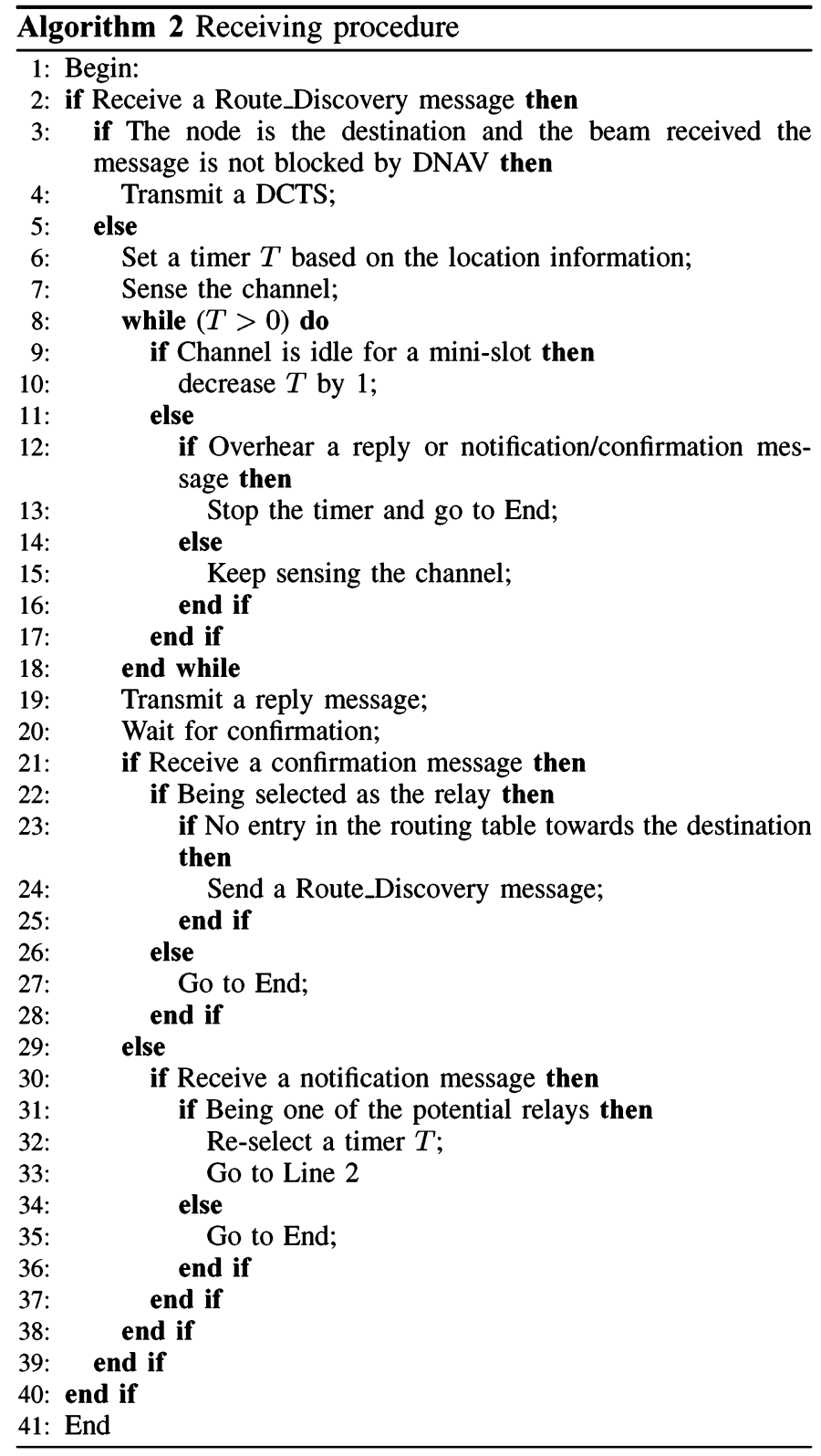

of new DRTS or new Route_Discovery messages by other nodes, the maximum contention backoff time $C T_{\max }$ (for the reply of the Route_Discovery message) should be less than a DIFS. We further divide the maximum backoff time into $W$ mini-slots and contending nodes select the corresponding timers based on their locations. How to set the backoff timer is critical for the protocol performance. To select an ideal relay, minimize the collisions of potential relays, and reduce the chance of Route_Discovery failure, we divide the region using the strategy shown in Fig. 4: nodes with distance $\left(d-\delta_{0} / 2, d+\right.$ $\delta_{0} / 2$ ) from the sender (zone 0 ) will set the backoff timer to be the minimal (one mini-slot); nodes within $\left(d_{i}-\delta_{i} / 2, d_{i}+\delta_{i} / 2\right)$ and $\left(d_{i}^{\prime}-\delta_{i}^{\prime} / 2, d_{i}^{\prime}+\delta_{i}^{\prime} / 2\right)$ will set the backoff to $i+1$ mini-slots, where $d_{i}=d+\delta_{0} / 2+\delta_{i} / 2+\sum_{k=1}^{i-1} \delta_{i}$ and $d_{i}^{\prime}=d-\delta_{0}^{\prime} / 2-\delta_{i}^{\prime} / 2-\sum_{k=1}^{i-1} \delta_{i}^{\prime}$ for $1 \leq i<W$.

The areas of different zones $\left(A_{i}\right)$ are:

$$
A_{0}=d \delta_{0} \theta
$$

$$
A_{i}=d_{i} \delta_{i} \theta+d_{i}^{\prime} \delta_{i}^{\prime} \theta \text { for } i>0 .
$$

The values of $\delta_{i}$ and $\delta_{i}^{\prime}$ are decided such that the area of zone $i+1\left(A_{i+1}\right)$ is twice as large as zone $i\left(A_{i}\right)$, for $i \geq 0$.

If more than one nodes send reply messages using the same backoff time, collision happens. In this case, the sender will transmit a collision notification message. For those nodes sending colliding reply messages, they will randomly select a backoff time in $[0, W]$, and repeat the contention procedure till the sender sends a confirmation message to notify who is the selected relay.

Note that in the first run of contention, the nodes within the same non-empty zone closest to zone 0 may send colliding reply messages. For a randomly distributed network, if the network density is high and zone 0 is not empty, the number of nodes in zone 0 is on average $1 /\left(2^{W}-1\right)$ of the total nodes in the transmission range of the Route_Discovery message only; if the network density is low and zones 0 to $i-1$ are empty, on average, the number of nodes in zone $i$ will not be much larger than one. For the second run, the nodes choosing the smallest value of backoff slots may collide and only them will enter the third run of contention. This design will ensure fast collision resolving, even with a very high node density. On the other hand, the next hop relay can be successfully found so long as there is at least one node in the transmission range of Route_Discovery.

\section{Route repairment}

If a path is broken due to user intermittency, channel impairments, or mobility, a node who cannot reach the next hop relay for a specified duration will initiate a route repairment procedure. Basically, it just removes the broken path from its routing table and broadcasts a new Route_Discovery message. If the node cannot find any relay toward the destination, it will report back to the upstream node which will broadcast a Route_Discovery message in directions other than the direction toward the one who reports the path broken.

\section{E. Effective concurrent transmissions}

To effectively explore spatial reuse, we need to allow concurrent transmissions if they do not introduce excessive interference to each other. Using the network in Fig. 2 as an example, $D_{s}$ is the carrier-sensing range. We should allow $S_{1}$ and $S_{4}$ transmit concurrently, while $S_{2}$ and $S_{3}$ should not transmit when $S_{1}$ is transmitting. However, when $S_{3}$ receives the DRTS from $S_{1}$, it only blocks the beam toward $S_{1}$, and it may transmit DRTS to $S_{4}$. Since $S_{4}$ 's beam toward $S_{3}$ is blocked by the DRTS from $S_{1}, S_{4}$ will not reply a DCTS. But the DRTS from $S_{3}$ can reach $S_{5}$ and block $S_{5}$ 's beam toward $S_{4}$. Thus, if $S_{4}$ sends DRTS after the failed DRTS from $S_{3}$, $S_{5}$ cannot reply a DCTS. Then, the channel between $S_{4}$ and $S_{5}$ is idle and wasted till the left beam of $S_{5}$ is unblocked. To solve the problem, it is desirable that $S_{3}$ does not transmit if it knows that the next hop's receiving beam is blocked. This is possible as $S_{3}$ has the location information of $S_{4}$, and it also has received the Route_Discovery and DRTS of $S_{1}$, so it knows the location of $S_{1}$. To implement the enhancement, 
before sending out an DRTS, the MAC protocol needs to check whether the receiver of the DRTS is possibly blocked or not.

\section{Performance Analysis ANd Parameter OPTIMIZATION}

For multi-hop wireless routing protocols, we are interested in maximizing the end-to-end flow throughput and minimizing the wireless resources used by the flow.

\section{A. Flow throughput}

First, we need to estimate the per-hop throughput. We assume that all nodes use the same power level to transmit since power adaptation in multi-hop wireless networks will introduce significant complexity to routing and MAC protocols. With mmWave technologies, nodes can adapt transmission data rate according to the SINR to efficiently utilize the wireless resources. To reveal the relationship of protocol performance and the parameters, we first assume that the hop distance is $d$ for all hops.

Denote $P$ the received signal power at reference distance $d_{0}=1 \mathrm{~m}$. Considering the path loss, the average received signal strength at distance $d \mathrm{~m}$ equals $P d^{-\alpha}$.

The interference level depends on the concurrent transmissions from other nodes. With the carrier-sensing based directional DCF MAC protocol, nodes within the carrier-sensing range $D_{s}$ of a sending node cannot transmit concurrently toward the sending node direction. As shown in the example in Fig. 2, $S_{1}, S_{2}$ and $S_{3}$ cannot transmit concurrently to $S_{2}, S_{3}$ and $S_{4}$, respectively, and $S_{1}$ and $S_{4}$ can transmit concurrently to $S_{2}$ and $S_{5}$, respectively. This is because for $S_{4}$ transmitting to $S_{5}$, the receiver $S_{5}$ is outside the DRTS transmission range from $S_{1}$ and it can reply a DCTS. In general, if the distance of each hop is $d, k=\max \left(2,\left\lfloor\frac{D_{s}}{d}\right\rfloor\right)$ nodes cannot transmit concurrently. The minimum of $k$ is two because the neighboring hops cannot transmit concurrently due to the half-duplex wireless transceiver design.

Now, we can estimate the maximum total interference to a node in multi-hop networks with directional antenna:

$$
I_{\max }=G_{0} P \sum_{i=1}^{\infty}\left(i D_{s}\right)^{-\alpha}=G_{0} P D_{s}^{-\alpha} \zeta(\alpha),
$$

where $G_{0}$ is the cross correlation between two transmissions and $\zeta()$ is the Riemann Zeta-function.

The per-hop throughput can be ensured if we consider the upper-bound of the interference:

$$
t h=\eta W \log _{2}\left(1+\frac{P d^{-\alpha}}{N+P D_{s}^{-\alpha} \zeta(\alpha)}\right),
$$

where $\eta$ is a system coefficient (a constant) related to the efficiency of the transceiver design.

If all hops in a path are un-saturated, given the per-hop throughput and the number of hops $k$ that cannot transmit concurrently, we can derive the flow throughput:

$$
\begin{aligned}
T H & =t h / k \\
& = \begin{cases}\frac{d}{D_{s}} \eta W \log _{2}\left(1+\frac{P d^{-\alpha}}{N+P D_{-}^{-\alpha} \zeta(\alpha)}\right) & \text { for } D_{s} \geq 2 d, \\
\frac{1}{2} \eta W \log _{2}\left(1+\frac{P d^{-\alpha}}{N+P D_{s}^{-\alpha} \zeta(\alpha)}\right) & \text { for } D_{s}<2 d .\end{cases}
\end{aligned}
$$

\section{B. Resources needed for each flow}

On the other hand, to efficiently utilize the wireless resources, we would like to minimize the resources used for each flow, so the network can support more flows without being saturated or overloaded. Since wireless resources have three dimensions, time, frequency, and space, the per-flow wireless resources needed are the product of them. For each hop, the space used for the transmission equals $\theta D_{s}^{2}$. Thus, the wireless resources used to transmit a unit of data over $k^{\prime}$ hops equal $\theta D_{s}^{2} W \frac{1}{t h} k^{\prime} \propto \frac{D_{s}^{2}}{t h \cdot d}$.

\section{Parameter optimization}

We can formulate two optimization problem, from the flow's point-of-view and from the network's point of view. For each flow, its main objective is to maximize its throughput, $\max T H$ (P1); for the network, the main objective is to minimize the resources needed for each flow, $\min \frac{D_{s}}{t h \cdot d}(\mathrm{P} 2)$.

If we can only adjust $d$, it is straight-forward to solve the above two optimization problems to find the best value for $d$ using (3) and (4). In addition, if $D_{s}$ is no less than $2 d$, the objective of maximizing flow throughput and the objective of minimizing resource needed for each flow will result in the same value of optimal $d$.

\section{MAC overhead consideration}

The above analysis use Shannon capacity to estimate the per-hop throughput, without considering the MAC protocol overheads. In this subsection, we first improve the analysis considering the MAC overheads, and further optimize the protocol accordingly.

A successful data packet transmission in each hop include the time to transmit a DRTS, a DCTS, the data packet, an ACK, three SIFSs and a DIFS.

$T=T_{D R T S}+T_{D C T S}+T_{d a t a}+T_{A C K}+3 T_{S I F S}+T_{D I F S}$.

The transmission times of DRTS, DCTS, and ACK are constant since they are transmitted at the lowest link rate (the base rate) to assure successful reception. The data transmission time is determined by the data payload $P l$ and the link rate (determined by SINR),

$$
T_{\text {data }}=\frac{P l}{\eta W \log _{2}\left(1+\frac{P d^{-\alpha}}{N+P D_{s}^{-\alpha} \zeta(\alpha)}\right)}+T_{\text {overhead }}
$$

where $T_{\text {overhead }}$ is the sum of the transmission times of PLCP preamble, the PHY header, and the MAC header and subheaders.

Thus, the per-hop throughput considering the MAC layer protocol overheads is given by

$$
t h=P l / T
$$

Substituting (7) in the objective function of (P1) and (P2), we can obtain the optimal $d$ to maximize flow throughput and minimize the resources used per flow, respectively. 
TABLE I

EXAMPLES OF THE INPUT PARAMETERS

\begin{tabular}{|c|c|}
\hline Parameters & Values \\
\hline Signal bandwidth $(W)$ & $1000 \mathrm{MHz}$ \\
\hline Transmission power $\left(P_{t}\right)$ & $0.1 \mathrm{~mW}$ \\
\hline Background noise $(N)$ & $-117 \mathrm{dBm} / \mathrm{MHz}$ \\
\hline Path loss exponent $(\alpha)$ & 2.17 \\
\hline Reference distance $\left(d_{r e f}\right)$ & $1 m$ \\
\hline Path loss at $d_{\text {ref }}\left(P L_{0}\right)$ & $68 \mathrm{~dB}$ \\
\hline Transceiver efficiency $(\eta)$ & 0.09842 \\
\hline Radiation angle $(\theta)$ & 30 degrees \\
\hline Payload & 8192 bits \\
\hline Header rate $\left(R_{h}\right)$ & $43 \mathrm{Mbps}$ \\
\hline Control rate $\left(R_{c}\right)$ & $58 \mathrm{Mbps}$ \\
\hline Data rate & less than or equal to $1 \mathrm{Gbps}$ \\
\hline Duration of the PLCP preamble $\left(T_{p r e}\right)$ & $1.383 \mu \mathrm{s}$ \\
\hline Duration of the PHY header $\left(T_{h d r}\right)$ & $0.395 \mu \mathrm{s}$ \\
\hline Number of aggregated packets $\left(n_{a g g}\right)$ & 1 \\
\hline Duration of MAC header and subheaders $\left(T_{m h}\right)$ & $\left(224+40 \cdot n_{a g g}\right)$ bits $/ R_{h}$ \\
\hline DRTS length & $160 \mathrm{bits} / R_{c}+T_{p r e}+T_{h d r}$ \\
\hline ACK length & 112 bits $/ R_{c}+T_{p r e}+T_{h d r}$ \\
\hline DCTS length & $4.0 \mu \mathrm{s}$ \\
\hline SIFS & $2.5 \mu \mathrm{s}$ \\
\hline DIFS & $34 \mu \mathrm{s}$ \\
\hline Duration of one mini-slot & $4.0 \mu \mathrm{s}$ \\
\hline $\begin{array}{l}\text { Carrier-sensing range }\left(D_{s}\right) \\
\text { Optimal hop distance }(d)\end{array}$ & $\begin{array}{l}18.16 \mathrm{~m} \\
6.053 \mathrm{~m}\end{array}$ \\
\hline
\end{tabular}

\section{NUMERICAL RESULTS}

The input parameters are listed in Table I. The signal bandwidth is $1000 \mathrm{MHz}$. The senders use the transmission power $P_{t}=0.1 \mathrm{~mW}$ and the background noise power is $-117 \mathrm{dBm} / \mathrm{MHz}$. Since mmWave signals attenuate fast in the air due to oxygen absorption and atmospheric attenuation, the path loss is measured as $68 \mathrm{~dB}$ at the reference distance $1 \mathrm{~m}$ with path loss exponent $\alpha=2.17$ [23]. The radiation angle of transceiver is set to $\theta=30$ degrees unless otherwise specified, and the transmitter and receiver antenna gains are $G_{t}=G_{r}$ $=360 / 30=12$. The parameter $\eta$ is set as 0.09842 so that the achievable data rate at the reference distance is one Gbps. The payload is 8192 bits. The data rate is less than or equal to one Gbps. The header rate is $43 \mathrm{Mbps}$ and the control rate is $58 \mathrm{Mbps}$ [22]. The duration of one mini-slot is $4.0 \mu \mathrm{s}$. It may be decreased with more sophisticated radios [24]. By using the values of the input parameters, we obtain the carrier-sensing range $D_{s}$ and optimal hop distance $d$ as $18.16 \mathrm{~m}$ and $6.053 \mathrm{~m}$, respectively.

Figs. 5 (a) and (b) compare flow throughput with and without considering the MAC overheads. First, the throughput is low when hop distance $d$ is small (close to $1 \mathrm{~m}$ ) or large (greater than $10 \mathrm{~m}$ ). Thus, both the opportunistic routing (prefer smallest hop distance) and the geographical routing (prefer largest hop distance within the transmission range) cannot perform well. If we choose the optimal $d$ as proposed, the flow throughput can be 2 to 3 times of those with the opportunistic and geographical routing protocols.

Second, changing the carrier-sensing range $D_{s}$ has little impact on maximizing throughput. However, with different value of $D_{s}$, the optimal value of $d$ is changed accordingly. The saw-teeth curves shown in the figure are due to the following two factors. When $\left\lfloor D_{s} / d\right\rfloor$ is smaller, more hops can transmit concurrently so the throughput can be increased

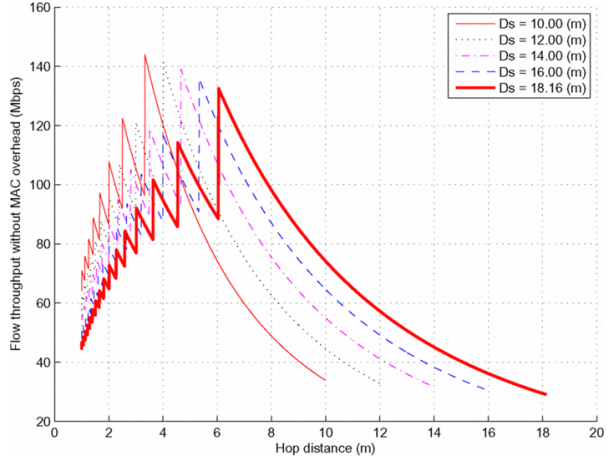

(a)

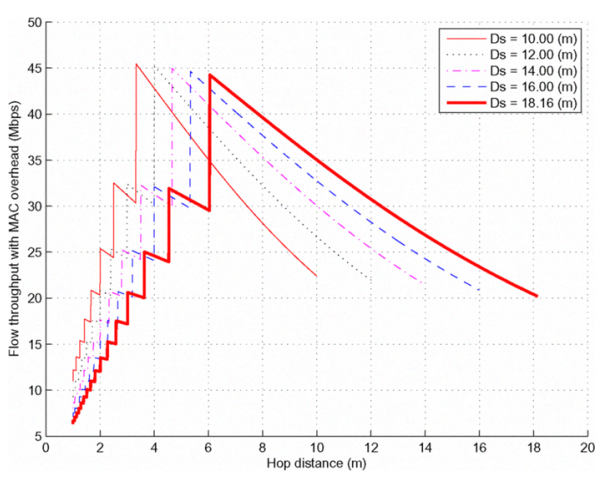

(b)

Fig. 5. Flow throughput with and without MAC overhead

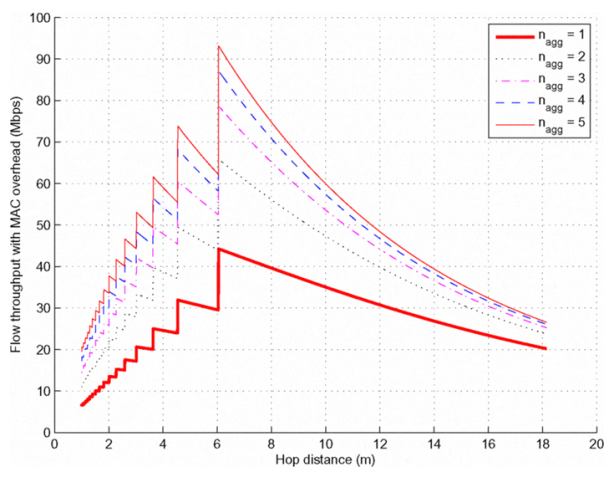

Fig. 6. Packet aggregation

till the throughput reaches its peak. On the other hand, with the same value of $\left\lfloor D_{s} / d\right\rfloor$, enlarging $d$ results in smaller SINR and data rate per hop, so the flow throughput decreases.

Third, the MAC layer overheads have a significant impact on the flow throughput, especially with high data rate mmWave communication links. The peak throughput drops more than $65 \%$ due to the overheads. Thus, it is desirable to use the packet aggregation technology. As shown in Fig. 6, by aggregating five packets into a link layer frame, the flow throughput is more than doubled at the peaks (with optimal $d$ ).

The flow throughput is also highly sensitive to the beam width of antenna and the channel path loss exponent, as shown in Figs. 7 and 8, respectively. When we have a larger beam 


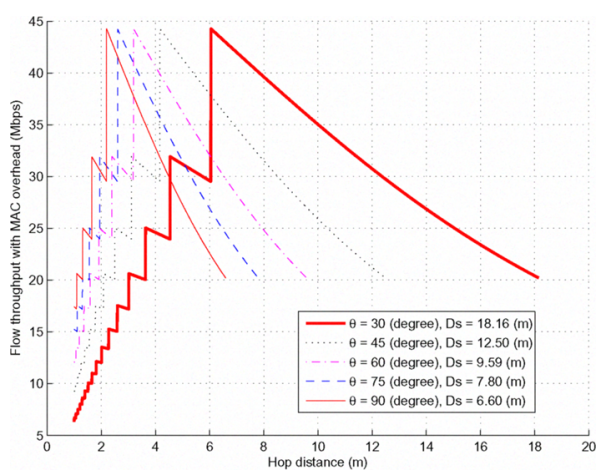

Fig. 7. Impact of antenna beam width

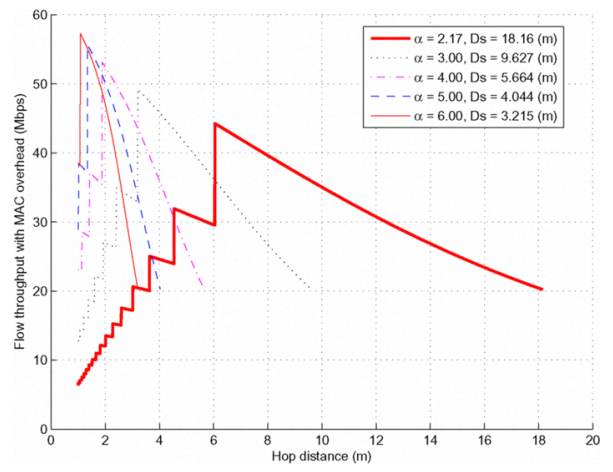

Fig. 8. Impact of path loss exponent

width $\theta$, the transmission distance is reduced, so does the optimal hop distance. The interesting observation is that the peak throughput does not change significantly w.r.t. $\theta$. This is because the increasing of $\theta$ will reduce both the signal strength and the interference. With lower interference and smaller value of $D_{s}$, more hops can transmit concurrently so the spatial reuse is higher.

As shown in Fig. 8, with a higher value of the path loss exponent $\alpha$, we can actually achieve slightly higher peak rate with smaller $D_{s}$ and $d$. This is because the larger value of $\alpha$, the less interference will be, and more concurrent transmissions are allowed.

In summary, no matter what are the values of path loss exponent and antenna beam width, choosing the value of hop distance appropriately can significantly improve the flow throughput and wireless resource utilization, compared with the existing opportunistic and geographic routing protocols.

\section{CONCLUSIONS}

We have proposed an Optimal Geographic Routing Protocol and an accompanying directional DCF MAC protocol for mmWave wireless networks. We have further derived the optimal hop distance to maximize the flow throughput and minimize the network resources used by the flow, considering the MAC overheads. Numerical results have demonstrated the effectiveness and efficiency of our proposed approach, which significantly outperforms the existing greedy geographic routing and opportunistic routing protocols.

\section{REFERENCES}

[1] I. Stojmenovic. Position-based routing in ad hoc networks. IEEE Communication Magazine, (7):128-134, Jul. 2002.

[2] Y. J. Kim, R. Govindan, B. Karp, and S. Shenker. On the pitfalls of geographic face routing. In roc. of DIALM-POMC'05, pages 34-43, 2005.

[3] H. Takagi and L. Kleinrock. Optimal transmission ranges for randomly distributed packet radio terminals. IEEE Trans. Commun., 32(3):246257, 1984.

[4] G. G. Finn. Routing and addressing problems in large metropolitan-scale internetworks. ISI res. rep. ISU/RR-87-180, Mar. 1987.

[5] A. Abdrabou and W. Zhuang. Statistical QoS routing for IEEE 802.11 multihop ad hoc networks. IEEE Transactions on Wireless Communications, 8(3):1542-1552, Mar. 2009.

[6] I. Stojmenovic and X. Lin. Loop-free hybrid single-path/flooding routing algorithms with guaranteed delivery for wireless networks. IEEE Trans. Paralle Dist. Sys., 12(10):1023-1032, 2001.

[7] B. Karp and H. T. Kung. GPSR: greedy perimeter stateless routing for wireless networks. In Proc. MobiCom'00, pages 243-254, 2000.

[8] F. Kuhn, R. Wattenhofer, and A. Zollinger. Worst-case optimal and average-case efficient geometric ad-hoc routing. In Proc. ACM MobiCom'03, pages 267-278, 2003.

[9] S. Subramanian, S. Shakkottai, and P. Gupta. On optimal geographic routing in wireless networks with holes and non-uniform traffic. In IEEE INFOCOM 2007, pages 1019-1027, May 2007.

[10] L. De Nardis, G. Giancola, and M.-G. Di Benedetto. A power-efficient routing metric for UWB wireless mobile networks. In Proc. IEEE VTC'03, pages 3105-3109, Oct. 2003.

[11] I. Stojmenovic and X. Lin. Power-aware localized routing in wireless networks. IEEE Transactions on Parallel and Distributed Systems, 12(11):1122-1133, 2001.

[12] P. Larsson. Selection diversity forwarding in a multihop packet radio network with fading channel and capture. In Proc. ACM MobiHoc'01, pages 279-282, 2001

[13] B. R. Hamilton and X. Ma. Noncooperative routing with cooperative diversity. In Proc. IEEE ICC'07, pages 4237-4242, Jun. 2007.

[14] S. Gezici, Z. Tian, G. B. Biannakis, H. Kobayashi, F. Molisch, H. V. Poor, and Z. Sahinoglu. Localization via UWB radios. IEEE Signal Processing Magazine, 22(4):70-84, Jul. 2005.

[15] W. Horie and Y. Sanada. Novel packet routing scheme based on location information for UWB ad-hoc network. pages 185-189, Nov. 2003.

[16] L. X. Cai, L. Cai, X. Shen, J.W. Mark, and Q. Zhang. Protocol design and optimization for multi-hop ultra-wideband networks. IEEE Trans. on Wireless Communications, 2009. to appear.

[17] L. De Nardis, G. Giancola, and M.-G. Di Benedetto. Power-aware design of MAC and routing for UWB networks. In Proc. Globecom'04, pages 235- 239, 2004

[18] L. X. Cai, L. Cai, X. Shen, and J.W. Mark. REX: a Randomized EXclusive region based scheduling scheme for mmWave WPANs with directional antenna. IEEE Trans. on Wireless Communications, 2009. to appear.

[19] L. X. Cai, L. Cai, X. Shen, and J. W. Mark. Resource management and QoS provisioning for IPTV over mmWave-based WPANs with directional antenna. ACM Mobile Networks and Applications (MONET, 14(2):210-219, 2009.

[20] IEEE 802.11 WG. Part 11: Wireless LAN Medium Access Control (MAC) and Physical Layer (PHY) specifications, Jun. 2003. 1999 ed., (R2003)

[21] R. Choudhury, X. Yang, R. Ramanathan, and N. Vaidya. Using directional antennas for medium access control in ad hoc networks. In Proc. of MOBICOM'02, pages 59-70, Mar. 2002.

[22] IEEE 802.15 WPAN Millimeter Wave Alternative PHY Task Group 3c (TG3c). Merged proposal: New PHY layer and enhancement of MAC for mmWave system proposal. Nov. 2007.

[23] S. Geng, J. Kivinen, X. Zhao, and P. Vainikainen. Millimeter-wave propagation channel characterization for short-range wireless communications. IEEE Trans. on Vehicular Technology, 58(1):3-13, Jan. 2009.

[24] D. P. Reed A. Bletsas, A. Khisti and A. Lippman. A simple cooperative diversity method based on network path selection. IEEE Journal on Selected Areas in Communications, 24(3):659-672, Mar. 2006. 